\title{
A Machine Learning Based Strategy to theOptimized Investment Portfolio
}

\author{
Author: Wei-JuChiu ${ }^{1}$; Shin-Jye Lee ${ }^{2}$; Ching-Hsun Tseng ${ }^{3}$ \\ Affiliation: Institute of Information Management, National Chiao Tung University, Taiwan ${ }^{1}$; \\ Institute of Management of Technology, National Chiao Tung University, Taiwan ${ }^{2}$;Department of \\ Computer Science, The University of Manchester, Manchester, $\mathrm{UK}^{3}$ \\ E-mail: tena10350506@gmail.com ${ }^{1}$;camhero@gmail.com ${ }^{2}$; $\underline{\text { hank131415go61@ gmail.com }{ }^{3}}$
}

DOI: 10.26821/IJSHRE.9.3.2021.9307

\begin{abstract}
Cash flow is the key leading companies to go further, like blood flowing in us. We need to face for those fixed cost and variable costs. But the source of the funds are always happened to be ignored by the founders in practical experience. They consider technique and creativity to be more essential. According to CB Insights's analysis on those failed startup companies, lacking of funds is the second reason that caused their companies to shut down. For startup companies, the way to increase investment funds is a significance issue. The way to use limited flow on investment will play an important role in how long they can keep a startup company alive. We would like to find the most optimized portfolio of affordable risk with comparatively high return to others. Using quantitative research and algorithms for machine learning to analyze which portfolio will be the best choice for those startup companies. Furthermore, we would dynamic adjust the factors in the algorithms for each period. Startups need to figure out how much they can earn in their business model. Processes on financing are always consuming more time than we assumed. Founders are always reserving enough time for turnover, and getting more source of funds to survive from the markets.
\end{abstract}

Keywords: Machine learning, Optimize investment portfolio

\section{INTRODUCTION}

Machine learning is booming in the financial robotics industry. Various wealth management companies use different models to find the portfolio with the highest return on investment. Technical analysis is based on price analysis. The internal principle is the strength and history of both buyers and sellers. A probabilistic analysis made on a repeat basis. In recent years, machine learning based on the disciplines of probability theory, statistics, approximation and so on has embodied its convenience and effectiveness in data analysis.

Machine learning is to make the computer system have human learning ability to realize artificial intelligence. It is widely used in various fields. This paper applies machine learning to the field of securities technology analysis, hoping to apply the post-learning analysis results to help new innovations. The company has achieved a steady flow of gold inflows.

Through the machine learning algorithm, the computer learns the rules in the historical price data of the securities, and then predicts the trading signals to realize the automated algorithm trading. Compared with the measurement analysis, the machine learning does not need to presuppose the specific analysis model, the machine learning model. The model parameters are self-adjusted according to the given training data, so that the trained parameters are the best description of the rules of the training data. The advantage is to get rid of the limitations of the analyst's understanding of the analytical model (such as the choice of different quantitative regression analysis models); the shortcoming is that it can not explain the impact of input variables on the output variables. However, in stock analysis and forecasting, especially in stock trading, we are more concerned with the accuracy of forecasting, which is why this paper applies machine learning methods to algorithm trading.

In nowadays, a large number of models have been developed. In the case of Exchange Traded Funds, four variables are usually taken into consideration, namely, the degree of risk preference confirmation, the position 
Volume 9 Issue 3 March 2021

of the boom, the reduction of asset correlation, and the timing inspection and adjustment of investment. In combination, there are other Investment Trust Fund Transactions that have also developed different models, such as the Mean Variance Optimization and the Black-Litterman Model used by the Franklin Investment Trust Fund Transaction. MVO is an excellent portfolio configuration framework, which can calculate the optimal allocation weight based on the estimated standard deviation, expected reward and correlation between each target category, while the Black-Litterman Model model combines market equilibrium compensation and managerial perspective. In order to arrive at the expected reward, it is possible to construct a more dispersed and intuitive portfolio.

While Tarabo Advisors uses Kelly Criterion's dynamic operations, the Kelly formula's goal is to maximize the long-term growth rate of investors. For long-term investors, this formula is more practical and rational than the commonly used modules, because Kelly criterion is from all. A portfolio of possible portfolios can maximize the long-term return on investment, while other examples include the FH Power X model of the Fuhhwa Trust Fund Transaction and the Macro Investment Advisor's self-accumulating big data model to re-use the CAPE Model principles.

Robot financing has sprung up in Taiwan, and all robots have advertised annualized rewards of more than $10 \%$ and $40 \%$. They can also bring investors to avoid Sino-US trade wars and stock market crashes. Robotic financial experts said that when investors choose robot financing, the key is to look at the core module and investment research team, and whether it is a machine learning mechanism. Therefore, the choice of the model will inevitably become the main reason for the accuracy of the forecast, and if such research results can be smoothly brought to the startup company, it will certainly bring a lot of benefits to the startup company.

\section{RELATED WORK}

\subsection{Investment Technology Analysis}

Lu Jialu. Lin Zhixuan [1] (2016) Rational investors often rely on technical analysis and basic analysis to increase profitability and reduce investment risk. The technical analysis is based on past statistical information, such as market supply and demand, market transaction price and transaction volume, and forecasts the development trend of securities prices as a reference for investors when buying and selling stocks. The basic analysis considers the overall investment environment, such as political, economic, and industrial cycles, as well as the analysis of the operating conditions, financial structure, and business development of individual companies, as the basis for investors to choose stocks. Since many investors conduct stock trading in practice, they will refer to technical analysis. However, if multiple technical indicators are used at the same time, investors often have no idea when the trading signals appear different.

\subsection{Use of Neural Networks}

Ruei Fu Je [2] (2016) A large number of literature studies have shown that support vector regression and artificial neural network have stronger generalization ability and higher prediction accuracy in processing stock data than other machine learning methods. Support vector regression is a variant of the support vector machine. It is a method of nonlinear regression. By adding the features of the data, construct one or more high-dimensional hyperplanes to classify the data points. A neural network is a parallel computing system that consists of many artificial neurons or nodes. Conceptually, a neural network is a neural network that mimics the human brain. The following model is established: each neuron represents a specific input/output function, and the input data is obtained by other neurons or the external environment, and processed by the arithmetic program.

\section{MODEL INTRODUCTION}

\subsection{Mean Variance Optimization (MVO)}

The goal of this law is to control the portfolio variance to a certain level that can be tolerated.The expected return rate of the portfolio is maximized, that is, the following nonlinear programming problems are solved.

$$
\begin{aligned}
& \underset{W}{\operatorname{Maximize}} W \bar{R}^{\prime} \\
& \text { subject to } W Q W^{\prime} \leq \sigma^{2} \\
& \qquad \sum_{i=1}^{n} w_{i}=1, \quad 0 \leq w_{i} \leq 1
\end{aligned}
$$

\subsection{XGBoost}

The name xgboost, though, actually refers to the engineering goal to push the limit of computations resources for boosted tree algorithms. Which is the reason why many people use xgboost.

It is an implementation of gradient boosting machines created by Tianqi Chen, now with contributions from many developers. It belongs to a broader collection of tools under the umbrella of the Distributed Machine 
Volume 9 Issue 3 March 2021

Learning Community or DMLC who are also the creators of the popular mxnet deep learning library.

\section{i) Model Features}

The implementation of the model supports the features of the scikitlearn and $R$ implementations, with new additions like regularization. Three main forms of gradient boosting are supported:

(1) Gradient Boosting algorithm also called gradient boosting machine including the learning rate.

(2) Stochastic Gradient Boosting with sub-sampling at the row, column and column per split levels.

(3) Regularized Gradient Boosting with both $L 1$ and $L 2$ regularization.

ii) System Features

The library provides a system for use in a range of computing environments, not least:

(1) Parallelization of tree construction using all of your CPU cores during training.

(2) Distributed Computing for training very large models using a cluster of machines.

(3) Out-of-Core Computing for very large datasets that don't fit into memory.

(4) Cache Optimization of data structures and algorithm to make best use of hardware.

\section{iii) Algorithm Features}

The implementation of the algorithm was engineered for efficiency of compute time and memory resources. A design goal was to make the best use of available resources to train the model. Some key algorithm implementation features include:
(1) Sparse Aware implementation with automatic handling of missing data values.

(2) Block Structure to support the parallelization of tree construction.

(3) Continued Training so that you can further boost an already fitted model on new data.

\section{CONCLUSION}

We hope that this time we can find the best investment portfolio by introducing Mean Varaiance Optimization into XGBoost's algorithm, which can help improve the accuracy of forecasting, improve the overall return on investment, and use this result to help startups benefit. To reduce their risk, the new company's funding sources are no longer just fundraising and venture capital companies, etc. and hope to help companies that have difficulties in the operation of the cash flow problem.

\section{REFERENCE}

[1] Lu Jialu and Lin Zhixuan, "Empirical Study on the Performance of Neural Network Portfolio Strategy," Volume 23, Issue 3, pp. 29-50, September 2016.

[2] [2] Ruei Fu Je, "Application of Machine Learning in Algorithmic Trading - Technical Analysis," Trading Valley, June 2016.

Wei Qiaoyi, "How to choose robot financing? Core brain, investment research team, learning mechanism is the key," ,Taipei, Commercial Times, July 2018. 